\title{
Wireless power at different scales
}

\author{
From submillimetre-sized devices to entire rooms, wireless power transfer is a valuable technology in a range \\ of settings.
}

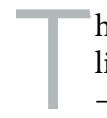

he appeal of wireless power transfer lies predominately in its convenience - it makes it easier to charge a phone, a laptop, an electric car. But the approach can also be used to unleash new capabilities. It can, for example, drive and control flexible microelectronic systems ${ }^{1}$. It can help create networks of sensors distributed across metamaterial textiles ${ }^{2}$. And perhaps of greatest value of all, it can allow sophisticated electronic devices to operate within the body.

Wireless power technology has already established itself as an option for cochlear and ocular implants ${ }^{3}$, and is now being used in a number of emerging, and increasingly intricate, biomedical devices. Last month in Nature Electronics, for instance, Arto Nurmikko and colleagues reported the development of wirelessly powered and networked microimplants ${ }^{4}$. These submillimetre-sized devices termed neurograins - have a gigahertz electromagnetic link to an external telecom hub, which affords bidirectional communication at the individual-device level. As a result, wirelessly networked ensembles of neurograins can be created that are capable of autonomous neural recording and stimulation.

Various techniques to build brainmachine interfaces are currently being explored, the most high profile of which being that of the company Neuralink, led by Elon Musk ${ }^{5}$. Such technology typically relies on monolithic arrays of microelectrodes. The neurograin system, with its ensembles of individual microdevices, could offer advantages in terms of placement flexibility and scaling. Nurmikko and colleagues who are based at Brown University, Baylor University, Seoul National University, the University of California San Diego, and Qualcomm Inc. - illustrate the potential of the approach by using 48 implanted neurograins to record neural activity in a rat model. They also suggest that the networking approach has the potential to be scaled to 770 neurograins.

Wireless power transfer is usually based on inductive coupling and, in particular, magnetically coupled resonant coils. The approach has a range of around a metre.

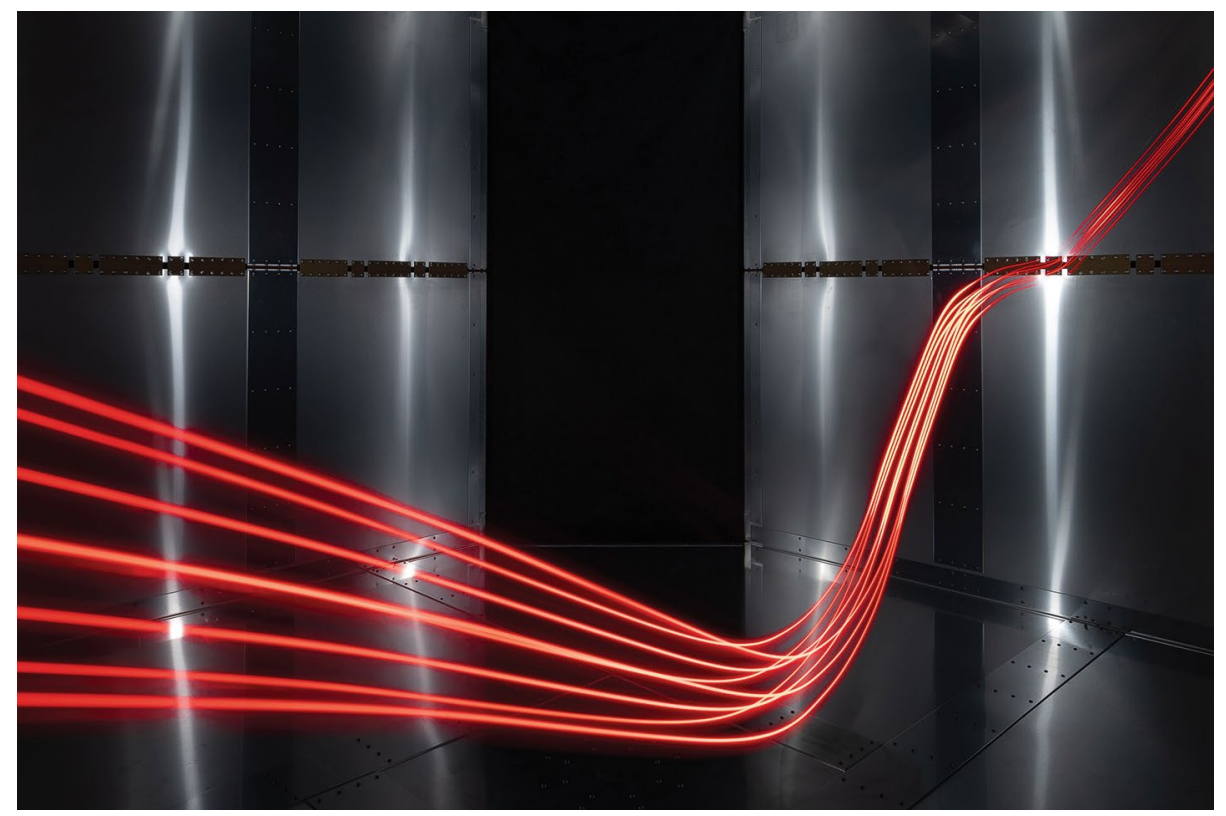

Photograph of a wireless power transfer test room built by Sasatani and colleagues. The long exposure of the photograph highlights the path that a wirelessly powered light-emitting diode (LED) took through the room. Credit: Takuya Sasatani and Yoshihiro Kawahara, The University of Tokyo.

This is often enough for implantable electronic devices. But if the focus is on the convenient charging of mobile electronic devices, it is not ideal. In an Article in this issue of Nature Electronics, Takuya Sasatani, Alanson Sample and Yoshihiro Kawahara now report room-scale wireless power transfer.

The researchers - who are based at the University of Tokyo and the University of Michigan - use a technique known as multimode quasistatic cavity resonance. It relies on a resonant structure that is composed of conductive surfaces and lumped capacitors, and can be built into the walls of a room. The approach generates two unique and widely distributed magnetic field patterns that can cover the entire volume of a room and can couple to small coil receivers attached to electrical devices.

Sasatani and colleagues highlight the capabilities of the approach by building a test room complete with everyday furnishings; lightbulbs, portable fans and smartphones could be wirelessly powered within the space. To maximize power transfer efficiency, the coil receivers should be orthogonal to the magnetic field, but an efficiency greater than $37.1 \%$ is still possible at any position within the room. Via simulations, the researchers also explore the safety of the approach and how much power would potentially be absorbed by a person in the room. The exposure levels remain comfortably within guidelines established by the Federal Communications Commission (FCC) and the Institute of Electrical and Electronics Engineers (IEEE).

Published online: 23 September 2021 https://doi.org/10.1038/s41928-021-00654-1

\footnotetext{
References

1. Bandari, V. K. et al. Nat. Electron. 3, 172-180 (2020)

2. Tian, X. et al. Nat. Electron. 2, 243-251 (2019).

. Khan, S. R. et al. Sensors 20, 3487 (2020)

4. Lee, J. et al. Nat. Electron. 4, 604-614 (2021).

5. Musk, E. et al. J. Med. Internet Res. 21, el6194 (2019).
} 